\title{
Correlation functions in unitary minimal Liouville gravity and Frobenius manifolds
}

\section{Belavin}

I.E. Tamm Department of Theoretical Physics, P.N. Lebedev Physical Institute, Leninsky prospect 53, 119991 Moscow, Russia

Department of Quantum Physics, Institute for Information Transmission Problems, Bolshoy Karetny per. 19, 127994 Moscow, Russia

Department of Theoretical Physics, National Research Nuclear University MEPhI, Kashirskoe shosse 31, 115409 Moscow, Russia

E-mail: belavin@lpi.ru

ABSTRACT: We continue to study minimal Liouville gravity (MLG) using a dual approach based on the idea that the MLG partition function is related to the tau function of the $A_{q}$ integrable hierarchy via the resonance transformations, which are in turn fixed by conformal selection rules. One of the main problems in this approach is to choose the solution of the Douglas string equation that is relevant for MLG. The appropriate solution was recently found using connection with the Frobenius manifolds. We use this solution to investigate three- and four-point correlators in the unitary MLG models. We find an agreement with the results of the original approach in the region of the parameters where both methods are applicable. In addition, we find that only part of the selection rules can be satisfied using the resonance transformations. The physical meaning of the nonzero correlators, which before coupling to Liouville gravity are forbidden by the selection rules, and also the modification of the dual formulation that takes this effect into account remains to be found.

Keywords: 2D Gravity, Conformal Field Models in String Theory, Matrix Models, Conformal and W Symmetry

ARXIV EPRINT: 1412.4245 


\section{Contents}

1 Introduction 1

2 Dual approach to MLG and Frobenius manifold structure 3

2.1 Flat coordinates 4

3 Resonance transformations $\quad 6$

3.1 First-order counterterms 6

$\begin{array}{lll}3.3 & \text { Second-order counterterms } & 7\end{array}$

4 Partition function and one-point correlators $\quad 8$

$\begin{array}{lll}4.2 & \text { Partition function } & 8\end{array}$

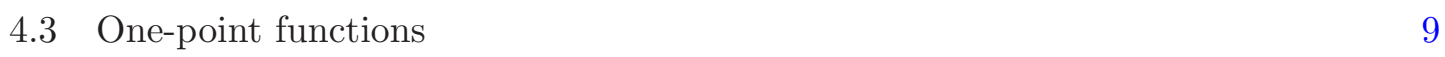

5 Two-point correlators $\quad 9$

6 Three-point correlators $\quad 10$

$\begin{array}{lll}6.1 & \text { Fusion rules and three-point functions } & 11\end{array}$

6.3 Comparing with MLG results 11

$\begin{array}{lll}\text { 6.3.1 Nonphysical region } & 12\end{array}$

$\begin{array}{lll}\text { 6.3.2 Physical region } & 13\end{array}$

7 Four-point correlators $\quad 14$

$\begin{array}{lll}8 & \text { Conclusions } & 17\end{array}$

$\begin{array}{ll}\text { A Details of the computations of the structure constants } & 18\end{array}$

$\begin{array}{ll}\text { B Some properties of the Jacobi polynomials } & 20\end{array}$

C Details of the calculation of the four-point correlator $\quad 21$

\section{Introduction}

The two-dimensional (2D) theory of Liouville gravity arises in the context of noncritical string theory [1]. This theory is called minimal Liouville gravity (MLG) in the case where the target space in the string sigma action or, equivalently, the matter sector in the worldsheet theory is represented by some minimal CFT model. Being a conformal theory, MLG can be studied by the standard methods of 2D CFT [2] (see, e.g., [3-6]). There is another, dual approach based on the natural geometric interpretation of MLG models as theories describing renormgroup fixed points of $2 \mathrm{D}$ quantum systems on fluctuating surfaces. 
Historically, the idea of fluctuating geometries first led to the development of the matrix-model (MM) approach to 2D gravity [7-16]. The coincidence of the spectra of gravitational dimensions in MLG and MM [17] gave reason to believe that the two approaches describe one theory. Unfortunately, the results of the MM and MLG approaches do not coincide on the level of correlation functions [18]; therefore, the MM approach is in fact concerned with some other class of $2 \mathrm{D}$ gravity models.

Strictly speaking, here by martix-models we mean a special class of MM proposed in [14] which is presumably connected with rational conformal theories coupled to $2 \mathrm{D}$ gravity. In this paper we do not consider interesting subject of non-rational theories of $2 \mathrm{D}$ quantum gravity and their matrix-models. However, we should note that studying non-rational case may help to answer some open conceptual questions of MLG theory. In particular, relaxing central charge parameter of the theory to the non-rational domain may help to trace out the change of the structure of the operator product expansion which occurs after coupling to Liouville gravity when the central charge takes rational values. This phenomenon is possibly related to the decoupling of non-degenerate fields from the spectrum. We should note also that in the case of non-rational models there exist some MM examples where the continuous interpretation is unambiguous (see, e.g., [19-21]). In this paper, however, we restrict ourself considering (motivated by Douglas MM [14]) dual description of genuine rational (minimal) CFT models coupled to Liouville gravity. Below we call this approach dual to distinguish it from other (matrix-model) approaches to 2D gravity.

It was first pointed out in [18] that a possible modification is related to the ambiguity in adding contact term interactions when defining MLG. Indeed, these contact terms are not controlled in the corresponding CFT theory and should be fixed by hand in order to define the integrated correlators. This ambiguity must be taken into account to reconcile the results of the dual and initial continuous approaches on the level of the correlation functions. Technically, this effect leads to the possible mixing of the Liouville coupling constants having compatible gravitational dimensions. This is called resonance transformations. The natural idea, proposed and further developed in $[18,22]$, is that this freedom must be fixed by MLG selection rules. On the sphere the selection rules contain the following requirements: vacuum expectation values of physical operators are absent, the two-point correlators are diagonal, and the conformal fusion rules are satiafied. In [22], this idea was applied to the series $M_{2,2 p+1}$ of MLG models, and the explicit form of the resonance transformation in terms of Legendre polynomials was found.

The new progress in developing the dual approach to MLG [23-25] is due to the connection between the Douglas string equation, ingtegrable Gelfand-Dikij hierarchies, and so-called $A_{q}$ Frobenius manifolds. This connection was used in [25] to analyze the unitary series $M_{q, q+1}$ of minimal models coupled to Liouville gravity. Based on properties of $A_{q}$ Frobenius manifolds, it was suggested that flat coordinates on the Frobenius manifold is most appropriate for analyzing the correlation functions. In particular, it was shown that the special solution of the Douglas string equation that is relevant for MLG has a simple form in flat coordinates. This idea was verified on the level of one- and two-point correlation numbers on the sphere, ${ }^{1}$ and it was shown that only using this solution allows satisfy the

\footnotetext{
${ }^{1}$ See [26] for some results of applying the dual approach to MLG in the torus case.
} 
basic requirements of the method that the so-called selection rules inherited by MLG from the conformal fusion rules of the CFT model in its matter sector be satisfied.

This paper is organized as follows. In section 2, we briefly review the dual approach to MLG and describe its connection with $A_{q}$ Frobenious manifolds. Section 3 is devoted to analyzing the resonance transformations. Sections 4, 5, 6, and 7 are respectively concerned with computations the one-, two-, three-, and four-point correlation functions. Section 8 contains concluding remarks. Some computation details are presented in appendices A, B, and $\mathrm{C}$.

\section{Dual approach to MLG and Frobenius manifold structure}

In this paper, we restrict our attention to the series of unitary models $M_{q, q+1}$ coupled to Liouville gravity in the spherical topology. In this case, the approach is formulated as follows. We introduce the so-called action $S$, which depends on $q-1$ parameters $u^{1}, u^{2}, \ldots, u^{q-1}$,

$$
S\left[u^{\alpha}\right]=\operatorname{res}_{y=\infty}\left(Q^{\frac{2 q+1}{q}}(y)+\sum_{1 \leq n \leq m \leq q-1} t_{m n}\left(\mu,\left\{\lambda_{k l}\right\}\right) Q^{\frac{(q+1) m-q n}{q}}(y)\right),
$$

where the polynomial

$$
Q(y)=y^{q}+u^{q-1} y^{q-2}+u^{q-2} y^{q-3}+\ldots+u^{1} y^{0} .
$$

The set of $\lambda_{k l}$ denotes the Liouville couplings, and the functions $t_{m n}(\mu, \lambda)$ are defined by the resonance transformations discussed in more detail below. The Duglas string equation [14] at genus zero [30] has the form

$$
\frac{\partial S}{\partial u^{\alpha}}=0, \quad \alpha=1, \ldots, q-1 .
$$

The main claim of the approach is that among the solutions of this system, there exists s special solution $\mathbf{u}_{*}=\left(u_{*}^{1}, u_{*}^{2}, \ldots, u_{*}^{q-1}\right)$ that can be used to construct the generating function of the correlators in MLG.

It was shown in [23] that the parameters $u^{\alpha}$ can be interpreted as the coordinates on the $(q-1)$-dimensional Frobenius manifold such that the metric in these coordinates is given by

$$
\left(\frac{\partial}{\partial u^{\alpha}}, \frac{\partial}{\partial u^{\beta}}\right)=-\operatorname{res}_{y=\infty} \frac{\frac{\partial Q(y)}{\partial u^{\alpha}} \frac{\partial Q(y)}{\partial u^{\beta}}}{\frac{d Q}{d y}} .
$$

To define the structure of the Frobenius manifold, we associated its points $\mathbf{u}$ with the $(q-1)$ dimensional Frobenius algebras $A_{q}(\mathbf{u})$ (depending on the parameters $u^{\alpha}$ ) such that $(2.4)$ represents a pairing of the algebra elements with the invariance property that $(a b, c)=$ $(a, b c)$ for arbitrary elements $a, b, c$ of the algebra. We recall that a finite-dimensional commutative and associative algebra with unity is called a Frobenius algebra if such an additional invariant pairing is defined for its elements. In the context of MLG, we deal with the $A_{q}$ Frobenius algebra that is the algebra of polynomials modulo the ideal generated by $Q^{\prime}(y)$. Using definition (2.4), all necessary properties of the Frobenius manifold (such as 
flatness of the metric, existence of the Frobenius potential, etc.) can be checked in the initial coordinates $u^{\alpha}$ [28]. This interpretation of the parameters $u^{\alpha}$ turns out to be very efficient. It was found in [25] that the problem of choosing the relevant solution can be solved by changing from the initial $\mathbf{u}$ to the flat coordinates $\mathbf{v}(\mathbf{u})$. More precisely, properties of the Frobenius manifolds were used to show that the relevant solution of the string equation $\mathbf{v}_{*}$ in the flat coordinates becomes $\mathbf{v}_{*}^{(0)}=\left(v_{* 1}, 0,0, \ldots\right)$ in the limit where the Liouville coupling constants are equal to zero. This solution is unique, i.e., only this solution gives the generating function (defined below) for which the necessary selection rules are satisfied on the level of one- and two-point correlators: it gives zero vacuum expectation values of the physical fields (except unity), and the two-point correlators are diagonal.

\subsection{Flat coordinates}

It follows from the properties of the Frobenius manifold that there exists a one-parametric deformation of the flat connection defined as follows [28]. The deformed coordinates ${ }^{2}$ are given by $\theta_{\alpha}(z)=\sum_{k=0}^{\infty} z^{k} \theta_{\alpha, k}$ such that $\theta_{\alpha}(0)=v_{\alpha}$. The transformation from the initial coordinates to the flat coordinates is defined by

$$
\theta_{\alpha, k}=-c_{\alpha, k} \underset{y=\infty}{\operatorname{res}} Q^{k+\frac{\alpha}{q}}(y)
$$

where

$$
c_{\alpha, k}^{-1}=\left(\frac{\alpha}{q}\right)_{k+1}
$$

and $(a)_{n}=\Gamma(a+n) / \Gamma(a)$ is the Pochhammer symbol. The flatness condition is equivalent to the recurrence relation

$$
\frac{\partial^{2} \theta_{\lambda}(z)}{\partial v^{\alpha} \partial v^{\beta}}=z C_{\alpha \beta}^{\gamma} \frac{\partial \theta_{\lambda}(z)}{\partial v^{\gamma}} .
$$

The corresponding deformation of the Levi-Civita connection is given by the modification of the Christoffel symbols

$$
\Gamma_{\alpha \beta}^{\gamma} \rightarrow \Gamma_{\alpha \beta}^{\gamma}+z C_{\alpha \beta}^{\gamma}
$$

The metric in the flat coordinates has the simple form

$$
\eta_{\alpha \beta}=-q \underset{y=\infty}{\operatorname{res}} \frac{\frac{\partial Q(y)}{\partial v^{\alpha}} \frac{\partial Q(y)}{\partial v^{\beta}}}{Q^{\prime}(y)}=\delta_{\alpha+\beta, q} .
$$

In particular, $v^{\alpha}=v_{q-\alpha}$.

It is highly nontrivial that deformed coordinates (2.5) can be regarded as local densities of the commuting Hamiltonians of the integrable Gelfand-Dikij hierarchies. An important consequence of this fact [23] is that the MLG generating function is just the logarithm of the tau function with the simple representation

$$
Z=\frac{1}{2} \int_{0}^{\mathbf{v}_{*}} C_{\alpha}^{\beta \gamma} \frac{\partial S}{\partial v^{\beta}} \frac{\partial S}{\partial v^{\gamma}} d v^{\alpha}
$$

\footnotetext{
${ }^{2}$ The deformed coordinates are flat coordinates with respect to the deformed connection defined below.
} 
Here, the upper limit $\mathbf{v}_{*}$ is the appropriate solution of the Douglas string equation

$$
\frac{\partial S\left(\mathbf{v}_{*}\right)}{\partial v^{\alpha}}=0, \quad \alpha=1, \ldots, q-1 .
$$

The integral in (2.10) is independent of the integration contour, which means that the integrand is a closed one-form. The structure constants of the Frobenius algebra in the flat coordinates $C_{\alpha}^{\beta \gamma}=C_{\alpha, q-\beta, q-\gamma}$ here are

$$
C_{\alpha \beta \gamma}=-q \operatorname{res} \frac{\frac{\partial Q(y)}{\partial v^{\alpha}} \frac{\partial Q(y)}{\partial v^{\beta}} \frac{\partial Q(y)}{\partial v^{\gamma}}}{Q^{\prime}(y)} .
$$

In particular, because $C_{1 \alpha \beta}=\eta_{\alpha \beta}$, we obtain

$$
C_{1}^{\alpha \beta}=\delta_{\alpha+\beta, q} \quad \text { and } \quad C_{\alpha}^{q-1, \beta}=\delta_{\alpha, \beta} .
$$

It follows from the definition of the Frobenius manifold that there exists a function $F(\mathbf{v})$ such that

$$
C_{\alpha \beta \gamma}(\mathbf{v})=\frac{\partial^{3} F(\mathbf{v})}{\partial v^{\alpha} \partial v^{\beta} \partial v^{\gamma}}, \quad \alpha, \beta, \gamma=1, \ldots, n .
$$

Before discussing the correlation functions, the following remark is in order. The structure constants in the flat coordinates are currently unknown, but we find that with the form of generating function (2.10) and the properties of the relevant solution $\mathbf{v}_{*}$ taken into account, the general expression for the structure constant is not needed for calculating the correlation function. Instead, we need the coefficients of the expansion

$$
C_{\alpha \beta \gamma}\left(v_{1}, v_{2}, v_{3}, \ldots\right)=C_{\alpha \beta \gamma}\left(v_{1}, 0,0, \ldots\right)+\sum_{\rho=1}^{q-1} v^{\rho} \partial_{\rho} C_{\alpha \beta \gamma}\left(v_{1}, 0,0, \ldots\right)+\ldots
$$

We are here interested in the three- and four-point correlation numbers. The necessary results for the first two terms of (2.15) are presented below. For a short representation, we introduce the function $\chi_{A, B}(x)=1$ if $x \in[A, B]$ and zero otherwise. In the zeroth order, the structure constant in the $\mathbf{v}$ coordinates on the solution of the string equation itself is

$$
C_{\alpha \beta \gamma}=\chi_{1, q-1}(\alpha+\beta-\gamma)\left(-\frac{v_{1}}{q}\right)^{\frac{\alpha+\beta+\gamma-q-1}{2}} \text { if } \frac{\alpha+\beta+\gamma-q-1}{2} \in \mathbb{N} \text {, else } 0 .
$$

The first derivative $\partial_{\rho} C_{\alpha \beta \gamma}$ is given by

$$
\begin{aligned}
\partial_{\rho} C_{\alpha \beta \gamma}= & {\left[(q-\rho) \chi_{1, \rho}(\alpha+\beta-\gamma)+\frac{2 q+\gamma-\alpha-\beta-\rho}{2} \chi_{\rho+2,2 q-\rho-2}(\alpha+\beta-\gamma)\right] \times(2 .} \\
& \times \frac{2 q-\alpha-\beta-\gamma-\rho}{2 q}\left(-\frac{v_{1}}{q}\right)^{\frac{\alpha+\beta+\gamma+\rho-2 q-2}{2}} \text { if } \frac{\alpha+\beta+\gamma+\rho-2 q-2}{2} \in \mathbb{N}, \text { else } 0
\end{aligned}
$$

In (2.16) and (2.17), $\mathbb{N}$ is the set of nonnegative integers, and we assume the ordering $\rho \geq \alpha \geq \beta \geq \gamma$. Because both tensors are symmetric, this information provides a complete answer. Some details of the derivation are given in appendix A. 


\section{Resonance transformations}

In the continuous approach the integrated correlators of MLG are defined up to so-called contact terms, which are not determined by CFT methods [22]. On the other hand, any change of contact terms is equivalent to resonance transformations of the coupling parameters. Explicitly, the resonance transformation has the form

$$
t_{m n}=\lambda_{m n}+\sum_{m_{1}, n_{1}}^{\delta_{m n}=\delta_{m_{1} n_{1}}+N} A_{N}^{\left(m_{1} n_{1}\right)} \mu^{N} \lambda_{m_{1} n_{1}}+\sum_{m_{1}, n_{1}, m_{2}, n_{2}}^{\delta_{m n}=\delta_{m_{1} n_{1}+\delta_{m_{2} n_{2}}+N}} A_{N}^{\left(m_{1} n_{1}, m_{2} n_{2}\right)} \mu^{N} \lambda_{m_{1} n_{1}} \lambda_{m_{2} n_{2}}+\ldots
$$

Here, $\mu$ and $\lambda_{m n}$ are the respective cosmological and Liouville coupling constants, $N$ is a nonnegative integer, each pair $\left(m_{i}, n_{i}\right)$ satisfies $^{3} 1 \leq n_{i} \leq m_{i} \leq q-1$, and the constants $A_{N}^{(i j)}, A_{N}^{(i j, k l)}, \ldots$ are the parameters of the resonance transformations. In (3.1), the gravitational dimensions are given by

$$
\delta_{m n}=\frac{2 q+1-|(q+1) m-q n|}{2 q},
$$

and hence $\lambda_{m n} \sim \mu^{\delta_{m n}}$.

By regrouping the terms, we can write action (2.1) in the form

$$
S=S^{(0)}+\sum_{m, n} \lambda_{m n} S^{(m n)}+\sum_{m_{1}, n_{1}, m_{2}, n_{2}} \lambda_{m_{1} n_{1}} \lambda_{m_{2} n_{2}} S^{\left(m_{1} n_{1}, m_{2} n_{2}\right)}+\ldots
$$

In what follows, we call the coefficients in expansion (3.3) counterterms. ${ }^{4}$

All required information concerning correlation functions is encoded in generating function (2.10). Namely, the correlation numbers are related to the coefficients in the coupling constant decomposition of the generating function

$$
Z=Z_{0}+\sum_{m_{1}, n_{1}} \lambda_{m_{1} n_{1}} Z_{m_{1} n_{1}}+\sum_{m_{1}, n_{1}, m_{2}, n_{2}} \lambda_{m_{1} n_{1}} \lambda_{m_{2} n_{2}} Z_{m_{1} n_{1}, m_{2}, n_{2}}+\ldots
$$

In what follows, we often use the short notation $\lambda_{m_{i}, n_{i}}=\lambda_{i}$ and $Z_{m_{i} n_{i}, m_{j} n_{j}, \ldots}=Z_{i j \ldots .}$.

\subsection{First-order counterterms}

Lemma 3.2. The first-order counterterms are given by

$$
S^{\left(m_{1} n_{1}\right)}=\underset{y=\infty}{\operatorname{res}} \sum_{N=0}^{\left\lfloor\frac{m-n}{2}\right\rfloor} A_{N}^{\left(m_{1} n_{1}\right)} \mu^{N} Q^{\frac{(q+1) m-q(n+2 N)}{q}}(y),
$$

where $m=m_{1}, n=n_{1}$, and $A_{N}^{\left(m_{1} n_{1}\right)}$ are the coefficients in the resonance relations of the coupling constants $\left(A_{0}^{\left(m_{1} n_{1}\right)}=1\right)$.

This statement follows trivially from (3.2).

\footnotetext{
${ }^{3}$ We always assume that this requirement is satisfied in what follows.

${ }^{4}$ We use this terminology from the renormalization theory because the additional terms in the action play exactly this role in responding to the shifts between the bare parameters $t_{m n}$ and the physical parameters $\lambda_{m n}$. According to this analogy, the selection rules for the correlators are regarded as renormalization conditions.
} 


\subsection{Second-order counterterms}

Taking (3.1) and (3.3) into account, we can derive the explicit form of $S^{\left(m_{1} n_{1}, m_{2} n_{2}\right)}$ from definition (2.1). The conditions on the pairs $\left(m_{1}, n_{1}\right)$ and $\left(m_{2}, n_{2}\right)$ for which there exist some $(m, n)$ in the Kac table of $M_{q, q+1}$ such the corresponding three gravitational dimensions are subject to the resonance balance are formulated below.

Lemma 3.4. The second-order counterterms are given by

$$
S^{\left(m_{1} n_{1}, m_{2} n_{2}\right)}=\underset{y=\infty}{\operatorname{res}} \sum_{N=0}^{\left\lfloor\frac{m-n}{2}\right\rfloor} A_{N}^{\left(m_{1} n_{1}, m_{2} n_{2}\right)} \mu^{N} Q^{\frac{(q+1) m-q(n+2 N)}{q}}(y),
$$

where

$$
\begin{array}{llll}
m=m_{1}+m_{2}-1, & n=n_{1}+n_{2}+1 & \text { for } & m_{1}+m_{2} \leq q, \\
m=m_{1}+m_{2}-q-1, & n=n_{1}+n_{2}-q & \text { for } & m_{1}+m_{2}>q .
\end{array}
$$

Proof. We seek solutions of the dimensional balance requirement

$$
\delta_{m_{1} n_{1}}+\delta_{m_{2} n_{2}}+N=\delta_{m n},
$$

where $N$ is a nonnegative integer and $n \leq m \leq q-1$. It is convenient to set $d=m-n$ and $d_{i}=m_{i}-n_{i}$. Explicitly, this requirement gives

$$
d_{1}+d_{2}+\frac{m_{1}+m_{2}}{q}=d+2 N+2+\frac{m+1}{q} .
$$

1. Let $m_{1}+m_{2}<q$. If $m+1=q$, then there is no solution because the noninteger factor $\frac{m_{1}+m_{2}}{q}$ cannot be compensated. If $m+1<q$, then

$$
\left\{\begin{array}{l}
d_{1}+d_{2}=d+2 N+2 \\
m_{1}+m_{2}=m+1
\end{array}\right.
$$

This gives $m=m_{1}+m_{2}-1$ and $n=n_{1}+n_{2}+1+2 N$.

2. Let $m_{1}+m_{2}=q$. If $m+1<q$, then there is no solution because the noninteger factor $\frac{m+1}{q}$ cannot be compensated. If $m+1=q$, then

$$
\left\{\begin{array}{l}
d_{1}+d_{2}+1=d+2 N+3, \\
m_{1}+m_{2}-q=0 .
\end{array}\right.
$$

This gives again $m=m_{1}+m_{2}-1$ and $n=n_{1}+n_{2}+1+2 N$, where $m_{1}+m_{2}=q$, and this case can hence be joined with the preceding case.

3. Let $q<m_{1}+m_{2} \leq 2 q-2$. If $m+1=q$, then there is no solution because the noninteger factor $\frac{m_{1}+m_{2}-q}{q}$ cannot be compensated. If $m+1<q$, then

$$
\left\{\begin{array}{l}
d_{1}+d_{2}+1=d+2 N+2, \\
m_{1}+m_{2}-q=m+1,
\end{array}\right.
$$

This gives $m=m_{1}+m_{2}-q-1$ and $n=n_{1}+n_{2}-q+2 N$. 


\section{Partition function and one-point correlators}

There is much evidence that the approach based on the Douglas string equation formulated in the preceding section provides an alternative description of MLG. A general proof of this statement is not yet available, but this conjecture can be checked by comparison with the results of computing directly in the framework of the continuous approach. Below, we provide further support of the hypothesis by performing some checks on the level of three- and four-point correlators. The last case is most important because the continuous approach here first requires nontrivial integration over the moduli space [4].

For convenience, we start our analysis of the correlation functions by presenting the results for the zero-, one-, and two-point correlators found in [25]. The consideration in this and in the following sections is based on the following statement.

Lemma 4.1. On the line $v_{i>1}=0$,

$$
\left\{\begin{aligned}
k-\text { even }: & \frac{\partial \theta_{\lambda, k}}{\partial v_{\alpha}}=\delta_{\lambda, \alpha} x_{\lambda, k}\left(-\frac{v_{1}}{q}\right)^{\frac{k}{2} q}, \\
k-\text { odd }: & \frac{\partial \theta_{\lambda, k}}{\partial v_{\alpha}}=\delta_{\lambda, q-\alpha} y_{\lambda, k}\left(-\frac{v_{1}}{q}\right)^{\frac{k-1}{2} q+\lambda},
\end{aligned}\right.
$$

where

$$
x_{\lambda, k}=\frac{1}{\left(\frac{\lambda}{q}\right)_{\frac{k}{2}}\left(\frac{k}{2}\right) !} \quad \text { and } \quad y_{\lambda, k}=-\frac{1}{\left(\frac{\lambda}{q}\right)_{\frac{k+1}{2}}\left(\frac{k-1}{2}\right) !} .
$$

In [25], this result was derived from recurrence relations (2.7).

\subsection{Partition function}

To define normalization independent quantities (or invariant cross ratios), we need the explicit form of the partition function

$$
Z_{0}=\frac{1}{2} \int_{0}^{\mathbf{v}_{*}^{(0)}} d v^{\gamma} C_{\gamma}^{\alpha \beta} \frac{\partial S^{(0)}}{\partial v^{\alpha}} \frac{\partial S^{(0)}}{\partial v^{\beta}}
$$

where $\mathbf{v}_{*}^{(0)}$ denotes the zeroth-order term of the expansion in coupling constants of the solution of the string equation.

To find it, we write the zeroth-order term of the expansion of the action explicitly in terms of deformed flat coordinates (2.5),

$$
S^{(0)}=\underset{y=\infty}{\operatorname{res}}\left[Q^{\frac{2 q+1}{q}}+\mu Q^{\frac{1}{q}}\right]=-\frac{\theta_{1,2}}{c_{1,2}}-\mu \frac{\theta_{1,0}}{c_{1,0}} .
$$

It can be seen from (4.1) that equations (2.11) for $\alpha<q-1$ are solved automatically by the ansatz $\mathbf{v}_{*}=\left(v_{* 1}, 0,0, \ldots\right)$, and we are hence left with the equation

$$
\frac{\partial S^{(0)}}{\partial v_{1}}=-\frac{1}{c_{1,2}} \frac{\partial \theta_{1,2}}{\partial v_{1}}-\frac{\mu}{c_{1,0}}=0
$$


which gives

$$
\mu=\frac{(1+q)(1+2 q)}{q}\left(-\frac{v_{* 1}}{q}\right)^{q} .
$$

This equation defines the zeroth-order term in the expansion of the appropriate solution of the string equation. The integration contour in (4.3) can be taken along the axis $v_{1}$ because on the line $\frac{\partial S^{(0)}}{\partial v_{k}}=0$ for $k>1$, we are left with the term containing $C_{q-1}^{q-1, q-1}=1$. An explicit calculation gives

$$
Z_{0}=\frac{(1+q)(1+2 q)}{q^{2 q+2}} v_{* 1}^{2 q+1}
$$

\subsection{One-point functions}

For the general one-point correlators, we obtain

$$
Z_{m n}=\int_{0}^{\mathbf{v}_{*}^{(0)}} d v^{\gamma} C_{\gamma}^{\alpha \beta} \frac{\partial S^{(0)}}{\partial v^{\alpha}} \frac{\partial S^{(m n)}}{\partial v^{\beta}} .
$$

Taking into account that $C_{\alpha}^{q-1, \beta}=\delta_{\alpha \beta}$ on the line $v_{k>1}=0$ and using the results in the preceding subsection, we more explicitly obtain

$$
Z_{m n}=\int_{0}^{\mathbf{v}_{*}^{(0)}} C_{q-1}^{q-1, \gamma} \frac{\partial S^{(0)}}{\partial v^{q-1}} \frac{\partial S^{(m n)}}{\partial v^{\gamma}} d v_{1}=\int_{0}^{\mathbf{v}_{*}^{(0)}} \frac{\partial S^{(0)}}{\partial v_{1}} \frac{\partial S^{(m n)}}{\partial v_{1}} d v_{1}
$$

Based on Lemma 4.1, we can conclude [25] that the one-point correlation numbers are equal to zero for all fields. ${ }^{5}$

\section{Two-point correlators}

An essential consistency requirement is that the two-point correlators be diagonal. This allows defining the first-order counterterms in the resonance transformations. Differentiating (2.10) twice, we obtain

$$
Z_{m_{1} n_{1}, m_{2} n_{2}}=\int_{0}^{\mathbf{v}_{*}^{(0)}} d v_{1} C_{q-1}^{\alpha \beta} \frac{\partial S^{\left(m_{1} n_{1}\right)}}{\partial v^{\alpha}} \frac{\partial S^{\left(m_{2} n_{2}\right)}}{\partial v^{\beta}}+\int_{0}^{\mathbf{v}_{*}^{(0)}} d v_{1} C_{q-1}^{\alpha \beta} \frac{\partial S^{(0)}}{\partial v^{\alpha}} \frac{\partial S^{\left(m_{1} n_{1}, m_{2} n_{2}\right)}}{\partial v^{\beta}} .
$$

The second term can be nonzero only for the correlators that have integer gravitational dimensions, which we do not consider. The first term gives

$$
Z_{m_{1} n_{1}, m_{2} n_{2}}=\sum_{\gamma=1}^{q-1}(-q)^{1-\gamma} \int_{0}^{\mathbf{v}_{*}^{(0)}} d v_{1} v_{1}^{\gamma-1} \frac{\partial S^{\left(m_{1} n_{1}\right)}}{\partial v_{\gamma}} \frac{\partial S^{\left(m_{2} n_{2}\right)}}{\partial v_{\gamma}} .
$$

It was found in [25] that for $v_{i>1}=0$,

$$
\frac{\partial S^{(m n)}}{\partial v_{\alpha}}\left(v_{1}\right)= \begin{cases}\delta_{m, \alpha} v_{* 1}^{\frac{m-n}{2} q}(-q)^{\frac{\alpha-1}{2}} N_{m n} P_{\frac{m-n}{2}}^{\left(0, \frac{m-q}{q}\right)}(t), & (m-n) \text { even } \\ \delta_{m, q-\alpha} v_{* 1}^{\frac{m-n-1}{2} q+m}(-q)^{\frac{\alpha-1}{2}} N_{m n}\left(\frac{1+t}{2}\right)^{\frac{m}{q}} P_{\frac{m-n-1}{2}}^{\left(0, \frac{m}{q}\right)}(t), & (m-n) \text { odd }\end{cases}
$$

\footnotetext{
${ }^{5}$ This statement holds up to usual indefiniteness related to the correlators having integer gravitational dimensions [22].
} 
where the new variable

$$
t=2\left(\frac{v_{1}}{v_{* 1}}\right)^{q}-1
$$

$P_{n}^{(0, b)}(t)$ are the Jacobi polynomials (see appendix B), and $N_{m n}$ denotes $t$-independent factors. Its explicit form is not relevant for our further consideration. With this result, the diagonality condition for the two-point correlators,

$$
Z_{m_{1} n_{1}, m_{2} n_{2}} \sim \delta_{m_{1}, m_{2}} \delta_{n_{1}, n_{2}},
$$

becomes equivalent to the orthogonality condition for the Jacobi polynomials. Calculating the diagonal two-point functions is straightforward,

$$
Z_{m n, m n}=\frac{N_{m n}^{2}}{(m-n) q+m} v_{* 1}^{(m-n) q+m} .
$$

\section{Three-point correlators}

One important change when we proceed to the level of three-point functions is that the derivative of the upper integration limit should be taken into account. Also for the first time, the contribution of the second-order counterterms arise on the level of three-point correlation functions. Using the same arguments based on the string equation and nonanalyticity requirements, we obtain the expression

$$
Z_{123}=\sum_{\sigma} \int_{0}^{\mathbf{v}_{*}^{(0)}} d v^{\gamma} C_{\gamma}^{\alpha \beta} \frac{\partial S^{(\sigma(1))}}{\partial v^{\alpha}} \frac{\partial S^{(\sigma(2) \sigma(3))}}{\partial v^{\beta}}+C_{\gamma}^{\alpha \beta} \frac{\partial \mathbf{v}_{*}^{\gamma}}{\partial \lambda_{3}} \frac{\partial S^{(1)}}{\partial v^{\alpha}} \frac{\partial S^{(2)}}{\partial v^{\beta}},
$$

where we replace the indices $\left(m_{i}, n_{i}\right)$ with index $i$ and the sum ranges permutations of the set $\{1,2,3\}$. In (6.1) and below, we always assume that the nonintegral part is evaluated on the solution of the string equation for all couplings equal to zero. In the rest of this paper, we use Latin indices exclusively in this sense, and the index zero means the zeroth-order term in the coupling constants expansion. The other terms in (6.16) disappear because they contain $\frac{\partial S^{(0)}}{\partial v^{\alpha}}\left(v_{*}\right)=0$. It follows from the string equation that

$$
\frac{\partial S^{(i)}}{\partial v^{\alpha}}+\frac{\partial^{2} S^{(0)}}{\partial v^{\alpha} \partial v^{\gamma}} \frac{\partial v_{*}^{\gamma}}{\partial \lambda_{i}}=0
$$

which gives

$$
\frac{\partial v_{*}^{\gamma}}{\partial \lambda_{i}}=T^{\gamma \beta} \frac{\partial S^{(i)}}{\partial v^{\beta}} .
$$

The inverse matrix $\left(T^{\alpha \gamma} M_{\gamma \beta}=\delta_{\beta}^{\alpha}\right)$

$$
M_{\alpha \beta}=-\frac{\partial^{2} S^{(0)}}{\partial v^{\alpha} \partial v^{\beta}},
$$

can be calculated using (4.4). The second term does not contribute to $M_{\alpha \beta}$, because $\frac{\partial^{2} \theta_{1,0}}{\partial v^{\alpha} \partial v^{\beta}}=\frac{\partial^{2} v_{1}}{\partial v^{\alpha} \partial v^{\beta}}=0$. For the first term, we use (4.1),

$$
M_{\alpha \beta}=\frac{1}{c_{1,2}} \frac{\partial^{2} \theta_{1,2}}{\partial v^{\alpha} \partial v^{\beta}}=\frac{1}{c_{1,2}} v_{1} C_{\alpha \beta}^{1} .
$$


With this result, we can easily find the inverse matrix $T^{\gamma \beta}$ :

$$
T^{\alpha \beta}=\frac{c_{1,2}}{v_{1}}\left(-\frac{v_{1}}{q}\right)^{1-\alpha} \delta_{\alpha, \beta} .
$$

The three-point function becomes

$$
Z_{123}=\sum_{\sigma} \int_{0}^{\mathbf{v}_{*}^{(0)}} d v^{\gamma} C_{\gamma}^{\alpha \beta} \frac{\partial S^{(\sigma(1))}}{\partial v^{\alpha}} \frac{\partial S^{(\sigma(2) \sigma(3))}}{\partial v^{\beta}}+C_{\rho}^{\alpha \beta} T^{\rho \gamma} \frac{\partial S^{(1)}}{\partial v^{\alpha}} \frac{\partial S^{(2)}}{\partial v^{\beta}} \frac{\partial S^{(3)}}{\partial v^{\gamma}},
$$

where the second term is evaluated on the solution of the string equation.

\subsection{Fusion rules and three-point functions}

We first formulate a useful consequence of the fusion rules for the three-point function in unitary minimal models $M_{q, q+1}$. We recall that the primary fields $\Phi_{m n}$ are labeled by $m=1, \ldots, q-1$ and $n \leq m$. For the three point function

$$
G=\left\langle\Phi_{m_{1} n_{1}} \Phi_{m_{2} n_{2}} \Phi_{m_{3} n_{3}}\right\rangle
$$

the fusion rules are satisfied, i.e., $G \neq 0$ if $P=\left(m_{1} n_{1}, m_{2} n_{2}, m_{3} n_{3}\right)$ or one of its reflection images generated by $\left(m_{i}, n_{i}\right) \rightarrow\left(q-m_{i}, q+1-n_{i}\right)$ belongs to the region

$$
F=\left\{\left(m_{1} n_{1}, m_{2} n_{2}, m_{3} n_{3}\right)\right\}
$$

such that for some permutation $(i, j, k)$ of the set $\{1,2,3\}$

$$
\left\{\begin{array}{c}
m_{k} \in\left[\left|m_{i}-m_{j}\right|+1: 2: \min \left(m_{i}+m_{j}-1,2 q-1-m_{i}-m_{j}\right)\right], \\
n_{k} \in\left[\left|n_{i}-n_{j}\right|+1: 2: \min \left(n_{i}+n_{j}-1,2 q+1-n_{i}-n_{j}\right)\right],
\end{array}\right.
$$

where $: 2:$ denotes step two. We can classify different cases with respect to the parities of $\sum_{i=1}^{3} m_{i}$ and $\sum_{i=1}^{3} n_{i}$. We can easily see the following consequence of the fusion rules for the unitary minimal models.

Proposition 6.2. In the case where both $\sum_{i} m_{i}$ and $\sum_{i} n_{i}$ are even, the three-point function $G=0$.

Indeed, for any choice of the reflection images of the fields, one of the parities $\sum_{i} m_{i}$ or $\sum_{i} n_{i}$ is even, which is forbidden by (6.10).

\subsection{Comparing with MLG results}

For the physically relevant nonanalytic correlators, there are two possibilities:

1. The fusion rules are not satisfied, the case nonphysical below. In this case, (6.7) must give zero whenever the resonance transformations permit it. This requirement allows defining the second-order counterterms $S^{(12)}$. 
2. The parameters $\left\{m_{i}, n_{i}\right\}$ of the three-point function $Z_{123}$ satisfy the fusion rules described above. We call this the physical region. In this case, (6.7) must give a result the same as the result derived in the continuous approach [4]. This can be achieved if the two conditions are satisfied:

(a) The integral part of (6.7) in this region is zero.

(b) The nonintegral part of (6.7) is nonzero and leads to the correct answer for the universal ratios.

Without loss of generality (interchanging the pairs if necessary), we can fix $2 \leq m_{1} \leq$ $m_{2} \leq m_{3}$. The general analysis of the three-point sector requires considering four different domains: all three fields are even, one field is odd, two fields are odd, all three fields are odd. Below, we analyze (1) and (2) in detail in the domain where all fields are even. Moreover, we impose the additional constraint

$$
m_{12} \leq m_{13} \leq m_{23} \leq q
$$

We note that the three-point functions are always nonanalytic in this domain. Indeed, the dimension

$$
\left[Z_{123}\right]=[Z]-\sum_{i=1}^{3} \delta_{i}=-1+\frac{m_{1}+m_{2}+m_{3}-1}{2 q}+\sum_{i=1}^{3} \frac{m_{i}-n_{i}}{2},
$$

where $m_{1}+m_{2}+m_{3} \leq 2 q-1$ and hence (6.12) is not integer.

\subsubsection{Nonphysical region}

Using the explicit form of the structure constants and the properties of first- and secondorder counterterms (5.3) and (3.8), we write the integral part IP of (6.7):

$$
\mathrm{IP}=\sum_{\sigma} \sum_{\gamma=1}^{q-1} \delta_{m_{i}, \gamma} \delta_{m_{j}+m_{k}-1, \gamma} \int_{0}^{\mathbf{v}_{*}^{(0)}} d v_{1}\left(-\frac{v_{1}}{q}\right)^{\gamma-1} \frac{\partial S^{(\sigma(i))}}{\partial v_{\gamma}} \frac{\partial S^{(\sigma(j), \sigma(k))}}{\partial v_{\gamma}}\left(v_{1}\right) .
$$

To find the second-order counterterm, we take

$$
m_{3}=m_{1}+m_{2}-1
$$

It can be seen that the terms with permutations disappear. Before we use change (5.4), it is convenient to express the second-order counterterms also in terms of the dimensionless functions $X^{\left(m_{1} n_{1}, m_{2} n_{2}\right)}(t)$ :

$$
\frac{\partial S^{\left(m_{1} n_{1}, m_{2} n_{2}\right)}}{\partial v_{m_{3}}}\left(v_{1}\right)=N_{1} N_{2} c_{1,2} 2^{-\frac{m_{3}}{2}}(-q)^{\frac{2 q-3+m_{3}}{2}} X^{\left(m_{1} n_{1}, m_{2} n_{2}\right)}(t) v_{* 1}^{\frac{m_{3}-n_{1}-n_{2}-1}{2} q} .
$$

The explicit form of the nonintegral part NIP in (6.7) is

$$
\mathrm{NIP}=N_{1} N_{2} N_{3} \frac{q^{q+1}}{(1+q)(1+2 q)} v_{1 *}^{\sum_{i}\left(\frac{m_{i}-n_{i}}{2} q+\frac{m_{i}}{2}\right)-\frac{1+2 q}{2}}
$$


Combining the integral and nonintegral parts, we obtain

$$
Z_{123}=N_{1} N_{2} N_{3} c_{1,2}(-q)^{q-2}\left[\int_{-1}^{1} d t(1+t)^{\frac{m_{3}-q}{q}} P_{\frac{m_{3}-n_{3}}{2}}^{\left(0, \frac{m_{3}-q}{q}\right)}(t) X^{\left(m_{1} n_{1}, m_{2} n_{2}\right)}(t)-1\right] .
$$

The degree of this polynomial $X^{\left(m_{1} n_{1}, m_{2} n_{2}\right)}(t)$ is

$$
\operatorname{deg} X^{\left(m_{1} n_{1}, m_{2} n_{2}\right)}=\frac{m_{1}-n_{1}+m_{2}-n_{2}-2}{2} .
$$

Because $m_{3}-n_{3}$ is even, $n_{3}-n_{1}-n_{2}$ should be odd:

$$
n_{3}=n_{1}+n_{2}+1+2 s, \quad s \in \mathbb{Z} .
$$

We consider the region $n_{3} \geq n_{1}+n_{2}$, where the three-point function should be zero according to the fusion rules. Because of the parity requirement, $n_{3}$ takes values from $n_{1}+n_{2}+1$ to $m_{3}$ with step 2 in this region. The degree of the Jacobi polynomial in (6.17) then changes from 0 to $\operatorname{deg} X^{(12)}$ with step 1 . Taking the completeness property of the Jacobi polynomials into account, we obtain

$$
X^{(12)}(t)=\sum_{k=0}^{\operatorname{deg} X^{(12)}}\left(q k+\frac{m_{1}+m_{2}-1}{2}\right) P_{k}^{\left(0, \frac{m_{1}+m_{2}-1}{q}-1\right)}(t),
$$

where the coefficients are fixed (using orthogonality (B.1)) from the requirement $Z_{123}=0$. Equation (B.3) can be used to obtain the representation ${ }^{6}$

$$
X^{(12)}(t)=q(1+t)^{1-\frac{m_{1}+m_{2}-1}{q}} \frac{d}{d t}\left[(1+t)^{\frac{m_{1}+m_{2}-1}{q}} P_{\frac{m_{1}-n_{1}+m_{2}-n_{2}}{2}-1}^{\left(0, \frac{m_{1}+m_{2}-1}{2}\right)}(t)\right] .
$$

\subsubsection{Physical region}

We must first ensure that if the fusion rules are satisfied, then the nonintegral part is nonzero. From (2.16), we find that NIP $\neq 0$ if

$$
m_{3}=m_{1}+m_{2}-1-2 s, \quad s=0,1,2, \ldots,
$$

and

$$
1 \leq q-m_{3}+m_{2}-m_{1} \leq q-1 .
$$

Taking (6.11) into account, we note that condition (6.22) is equivalent to $m_{3} \leq \min \left(m_{1}+\right.$ $\left.m_{2}-1,2 q-1-m_{1}-m_{2}\right)$. The right condition in (6.23) gives $m_{2}-m_{1}+1 \leq m_{3}$, while the left condition is satisfied because $q-m_{3}+m_{2}-m_{1} \geq q-m_{3}$. Hence, if the fusion rules are satisfied, then the nonintegral part in (6.7) is nonzero.

The second step is to verify that the integral part in (6.7) is absent when the fusion rules are satisfied. It follows from Lemma 3.4 that $\mathrm{IP}=0$ if $m_{3} \neq m_{1}+m_{2}-1$. Hence, all we need to verify is the case $m_{3}=m_{1}+m_{2}-1$. From the fusion rules, we have

$$
\left|n_{1}-n_{2}\right|+1 \leq n_{3} \leq n_{1}+n_{2}-1
$$

\footnotetext{
${ }^{6}$ We note that according to the results in Lemma 3.4, $m_{1}+m_{2}-n_{1}-n_{2} \geq 2$.
} 
where the choice of the right-hand side takes $n_{i} \leq m_{i}$ and (6.11) into account. From (6.18), we derive

$$
\operatorname{deg} X^{\left(m_{1} n_{1}, m_{2} n_{2}\right)}(t)<\operatorname{deg} P_{\frac{m_{3}-n_{3}}{2}}^{\left(0, \frac{m_{3}-q}{q}\right)}(t),
$$

and using the completeness and orthogonality properties of Jacobi polynomials, we conclude that the integral part is equal to zero.

The final step is to check the result for three-point universal ratio. For general $(q, p)[4]$, it has the form

$$
\frac{\left\langle\left\langle O_{1} O_{2} O_{3}\right\rangle\right\rangle^{2}}{\prod_{i=1}^{3}\left\langle\left\langle O_{i}\right\rangle\right\rangle}=\frac{\prod_{i=1}^{3}\left|p m_{i}-q n_{i}\right|}{p(p+q)(p-q)},
$$

where $\langle\langle\ldots\rangle\rangle=\frac{\langle\ldots\rangle}{\langle 1\rangle}$. For $p=q+1$, this expression coincides with the three-point universal ratio obtained in the dual approach

$$
\frac{\left(Z_{123}\right)^{2} Z_{0}}{Z_{11} Z_{22} Z_{33}}=\frac{\prod_{k=1}^{3}\left((q+1) m_{i}-q n_{i}\right)}{(1+q)(1+2 q)}
$$

Here, we use (6.16), (5.6), and (4.7).

\section{Four-point correlators}

In this section, we perform some checks for the four-point correlators. We are mainly focused on the following puzzle. From the preceding consideration, it might appear that the role of the parameters $n_{i}$ is somewhat suppressed with respect to the role of the parameters $m_{i}$. Indeed, apart from the normalization, it seems that all they do is choose the region according to the parities $m_{i}-n_{i}$, and the values of $n_{i}$ seem irrelevant. Below, we demonstrate how the balance between $m_{i}$ and $n_{i}$ is recovered on the four-point level.

The general expression for the four-point correlator is

$$
Z_{1234}=Z_{1234}^{\mathrm{NIP}}+Z_{1234}^{\mathrm{IP}}
$$

where $^{7}$

$$
\begin{aligned}
Z_{1234}^{\mathrm{NIP}}= & \frac{\partial^{2} v_{*}^{\gamma}}{\partial \lambda_{3} \partial \lambda_{4}} C_{\gamma}^{\alpha \beta} S_{\alpha}^{(2)} S_{\beta}^{(1)}+\frac{\partial v_{*}^{\gamma}}{\partial \lambda_{3}} \frac{\partial v_{*}^{\delta}}{\partial \lambda_{4}} C_{\gamma}^{\alpha \beta}\left(S_{\beta}^{(1)} S_{\alpha \delta}^{(2)}+S_{\beta \delta}^{(1)} S_{\alpha}^{(2)}\right)+ \\
& +\frac{\partial v_{*}^{\gamma}}{\partial \lambda_{3}} \frac{\partial C_{\gamma}^{\alpha \beta}}{\partial \lambda_{4}} S_{\alpha}^{(2)} S_{\beta}^{(1)}+\frac{\partial v_{*}^{\gamma}}{\partial \lambda_{4}} C_{\gamma}^{\alpha \beta}\left(S_{\alpha}^{(12)} S_{\beta}^{(3)}+\text { permutations }\right)
\end{aligned}
$$

and

$$
Z_{1234}^{\mathrm{IP}}=\frac{1}{2} \int_{0}^{v_{* 1}} d v^{\gamma} C_{\gamma}^{\alpha \beta}\left(S_{\alpha}^{(1234)} S_{\beta}^{(0)}+S_{\alpha}^{(123)} S_{\beta}^{(4)}+S_{\alpha}^{(12)} S_{\beta}^{(34)}+\text { permutations }\right) .
$$

In (7.2) and (7.3), we use $S_{\alpha}^{(i \ldots)}=\frac{\partial S^{(i \ldots)}}{\partial v^{\alpha}}$ and $S_{\alpha \beta}^{(i \ldots)}=\frac{\partial^{2} S^{(i \ldots)}}{\partial v^{\alpha} \partial v^{\beta}}$. According to the general pattern sketched in 6.3 , we assume that the integral part $Z_{1234}^{\mathrm{IP}}=0$ in the region where the fusion rules are satisfied.

\footnotetext{
${ }^{7}$ To make our formulas less cumbersome, we just remember that the resulting expression is to be calculated on the solution of the string equation. Therefore, in particular, there is no term containing $S_{\alpha}^{(0)}$ in the nonintegral part.
} 
We consider the case where there are no higher-order counterterms starting from the second order and the first derivatives of the structure constant are zero, ${ }^{8}$ and the term with

$$
\frac{\partial C_{\gamma}^{\alpha \beta}}{\partial \lambda_{i}}=T^{\rho \eta} \partial_{\rho} C_{\gamma}^{\alpha \beta} S_{\eta}^{i}
$$

is hence absent. Under these assumptions, only the first two terms in (7.2) survive:

$$
Z_{1234}=\frac{\partial^{2} v_{*}^{\gamma}}{\partial \lambda_{3} \partial \lambda_{4}} C_{\gamma}^{\alpha \beta} S_{\alpha}^{(2)} S_{\beta}^{(1)}+\frac{\partial v_{*}^{\gamma}}{\partial \lambda_{3}} \frac{\partial v_{*}^{\delta}}{\partial \lambda_{4}} C_{\gamma}^{\alpha \beta}\left(S_{\beta}^{(1)} S_{\alpha \delta}^{(2)}+S_{\beta \delta}^{(1)} S_{\alpha}^{(2)}\right) .
$$

Similar to (6.3), we can find second derivatives of the solution of the string equation

$$
\frac{\partial^{2} v_{*}^{\gamma}}{\partial \lambda_{i} \partial \lambda_{j}}=T^{\gamma \rho} T^{\sigma \chi}\left(S_{\rho \sigma}^{(i)} S_{\chi}^{(j)}+S_{\rho \sigma}^{(j)} S_{\chi}^{(i)}+T^{\eta \delta} S_{\rho \sigma \eta}^{(0)} S_{\chi}^{(i)} S_{\delta}^{(j)}\right) .
$$

Combining all together, we obtain the structure of the four-point correlator:

$$
\begin{aligned}
Z_{1234}= & T^{\gamma \rho} T^{\sigma \chi} C_{\gamma}^{\alpha \beta} S_{\alpha}^{(1)} S_{\beta}^{(2)}\left(S_{\rho \sigma}^{(3)} S_{\chi}^{(4)}+S_{\chi}^{(3)} S_{\rho \sigma}^{(4)}\right)+ \\
& T^{\gamma \mu} T^{\delta \nu} C_{\gamma}^{\alpha \beta}\left(S_{\beta}^{(1)} S_{\alpha \delta}^{(2)}+S_{\beta \delta}^{(1)} S_{\alpha}^{(2)}\right) S_{\mu}^{(3)} S_{\nu}^{(4)}+ \\
& T^{\gamma \rho} T^{\sigma \chi} T^{\eta \delta} C_{\gamma}^{\alpha \beta} S_{\rho \sigma \eta}^{(0)} S_{\alpha}^{(1)} S_{\beta}^{(2)} S_{\chi}^{(3)} S_{\delta}^{(4)}
\end{aligned}
$$

Even without the contribution of the higher counterterms, we see a few new objects in this expression that require additional calculations. The details of the calculations are in appendix C. For the third derivative $S_{\rho \sigma \eta}^{(0)}$, we obtain

$$
S_{\beta \rho \eta}^{(0)}=\frac{q-\beta-\rho-\eta-1}{2 c_{1,2}} C_{\beta \rho \eta},
$$

and the second derivative $S_{\alpha \beta}^{(i)}$ is given by

$$
S_{\alpha \beta}^{(m n)}=\frac{1}{2} C_{\alpha \beta}^{m}\left(-\frac{v_{1}}{q}\right)^{m+1-q} R_{m n},
$$

where there is no summation over $m$ and

$$
R_{m n}=\frac{1}{2}(m-n)(2 m+q(m-n)) .
$$

In the calculation, we find the three basic structures

$$
\begin{aligned}
& F_{1}(\chi, \xi, \mu, \nu)=\sum_{\gamma=1}^{q-1} \theta(\gamma, \chi, \xi) \theta(\gamma, \mu, \nu), \\
& F_{2}(\chi, \xi, \mu, \nu)=\sum_{\gamma=1}^{q-1} \theta(\gamma, \chi, \xi) \theta(q-\gamma, \mu, \nu), \\
& F_{3}(\chi, \xi, \mu, \nu)=\sum_{\gamma=1}^{q-1} \gamma \theta(\gamma, \chi, \xi) \theta(\gamma, \mu, \nu) .
\end{aligned}
$$

\footnotetext{
${ }^{8}$ This requirement gives, of course, additional restrictions on the parameters $m_{i}, n_{i}$ of the four-point correlator.
} 
Here, each $\theta(\alpha, \beta, \gamma)$ is related to one of the structure constants in $(7.7),(7.8)$, or (7.9). We recall that it is explicitly defined as a symmetric tensor such that $\theta(\alpha, \beta, \gamma)=\chi_{1, q-1}(\alpha+$ $\beta-\gamma$ ) if $\alpha \geq \beta \geq \gamma$. After some computations (the details can be found in appendix C), we obtain

$$
\begin{aligned}
F_{1}= & \frac{1}{4}(2 q-(|\chi-\xi|+|\mu-\nu|+|| \chi-\xi|-| \mu-\nu||+|\chi+\xi-q|+ \\
& +|\mu+\nu-q|+|| \chi+\xi-q|-| \mu+\nu-q||)), \\
F_{2}= & \frac{1}{4}(2 q-(|\chi-\xi|+|\mu+\nu-q|+|| \mu+\nu-q|-| \chi-\xi||+ \\
& +|\chi+\xi-q|+|\mu-\nu|+|| \chi+\xi-q|-| \mu-\nu||)), \\
F_{3}= & F_{1} \frac{1}{4}(2 q-(|\chi-\xi|+|\mu-\nu|+|| \chi-\xi|-| \mu-\nu||)+ \\
& +|\chi+\xi-q|+|\mu+\nu-q|+|| \chi+\xi-q|-| \mu+\nu-q||) .
\end{aligned}
$$

In terms of these functions with the convention $R_{i}=R_{m_{i} n_{i}}$, the four-point correlator is

$$
\begin{aligned}
Z_{1234}= & N\left[R_{1} F_{2}\left(q-m_{4}, q-m_{3}, q-m_{1}, m_{2}\right)+R_{2} F_{2}\left(q-m_{4}, q-m_{3}, q-m_{2}, m_{1}\right)+\right. \\
& R_{3} F_{1}\left(q-m_{3}, m_{1}, m_{2}, q-m_{4}\right)+R_{4} F_{1}\left(q-m_{3}, m_{1}, m_{2}, q-m_{4}\right)+ \\
& \left.+\left(m_{1}+m_{2}-q-1\right) F_{1}\left(m_{1}, m_{2}, q-m_{3}, q-m_{4}\right)-F_{3}\left(m_{1}, m_{2}, q-m_{3}, q-m_{4}\right)\right] .
\end{aligned}
$$

Here, the first four terms come from the terms in (7.7) containing second derivatives of the first-order counterterms, and the last two terms come from the term with the third derivative of the action $S^{(0)}$. The overall normalization factor ${ }^{9}$ is

$$
N=\frac{c_{1,2}^{2}}{2}\left(-\frac{1}{q}\right)^{2-2 q+\frac{m_{1}+m_{2}+m_{3}+m_{4}}{2}} .
$$

For the four-point correlators, the universal ratio, which is independent of the normalizations, can be constructed from (7.17), (5.6), and (4.7):

$$
\left\langle\left\langle O_{m_{1} n_{1}} O_{m_{2} n_{2}} O_{m_{3} n_{3}} O_{m_{4} n_{4}}\right\rangle\right\rangle_{\text {norm }}=\frac{Z_{m_{1} n_{1}, m_{2} n_{2}, m_{3} n_{3}, m_{4} n_{4}} Z_{0}}{\left(\prod_{i=1}^{4} Z_{m_{i} n_{i}, m_{i} n_{i}}\right)^{\frac{1}{2}}} .
$$

This result is to be compared with the four-point correlator in MLG calculated in [4] using the standard continuous approach,

$$
\begin{aligned}
& \frac{\left\langle\left\langle O_{m_{1} n_{1}} O_{m_{2} n_{2}} O_{m_{3} n_{3}} O_{m_{4} n_{4}}\right\rangle\right\rangle}{\left(\prod_{i=1}^{4}\left\langle\left\langle O_{m_{i}, n_{i}}^{2}\right\rangle\right\rangle\right)^{\frac{1}{2}}}=\frac{\prod_{i=1}^{4}\left|m_{i} p-n_{i} q\right|}{2 p(p+q)(p-q)} \times \\
& \times\left[\sum_{i=2}^{4} \sum_{r=-\left(m_{1}-1\right)}^{m_{1}-1} \sum_{t=-\left(n_{1}-1\right)}^{n_{1}-1}\left|\left(m_{i}-r\right) p-\left(n_{i}-t\right) q\right|-m_{1} n_{1}\left(m_{1} p+n_{1} q\right)\right],
\end{aligned}
$$

where $p=q+1$ for the unitary series.

\footnotetext{
${ }^{9}$ Here we suppress usual dimensional factor $v_{* 1}^{\delta_{1234}}$.
} 
One further point should be noted. Expression (7.20) also has some restrictions that should be taken into account. In particular, the active field for which the operator product expansion is used must have the smallest product $m_{i} n_{i}$ among the four pairs. Moreover, the number of the conformal blocks [4] must be equal to this number. This restriction together with the requirement that higher counterterms be absent can be satisfied for general $q$ if we consider symmetric correlation functions of the form $\left\langle\left\langle O_{m n}^{4}\right\rangle\right\rangle_{\text {norm. }}$. We note that in this case, expression (7.7) (only partially symmetric) becomes completely symmetric with respect to the permutations of the fields, as it should.

To give some reference points, we quote a few results for the gravitational Ising, tricritical Ising, and three-state Potts models corresponding to $M_{3,4}, M_{4,5}$, and $M_{5,6}$. Two nontrivial completely symmetric four-point correlators are the correlator of four spin-density operators $\sigma=\Phi_{12}$ and the correlator of four energy-density operators $\epsilon=\Phi_{13}$ dressed by the appropriate Liouville exponential fields. We find that the two expressions (7.19) and (7.20) give the same results:

\begin{tabular}{|c|c|c|}
\hline & $\langle\langle\sigma \sigma \sigma \sigma\rangle\rangle_{\text {norm }}$ & $\langle\langle\epsilon \epsilon \epsilon \epsilon\rangle\rangle_{\text {norm }}$ \\
\hline$M_{3,4}$ & $-\frac{1}{7}$ & $\frac{75}{11}$ \\
$M_{4,5}$ & $-\frac{1}{5}$ & $\frac{49}{5}$ \\
$M_{5,6}$ & $-\frac{8}{33}$ & $\frac{162}{11}$ \\
\hline
\end{tabular}

We note that to obtain these results, we must, as we fixed in the very beginning, take pairs $(m, n)$ with $m \geq n$ in (7.19) and their reflection images $(q-m, p-n)$ in (7.20).

\section{Conclusions}

We have partially analyzed the three- and four-point correlation functions using the dual approach to MLG. In the domain where the fusion rules are satisfied, we found agreement with the results of the continuous approach.

According to the results in Lemma 3.2 and Lemma 3.4, a rigorous analysis requires much more systematic classification. For three-point functions (6.1), for example, there exist four possible regions, depending on which interval contains the parameter $q$, for example, $m_{12} \leq q \leq m_{23}$, etc., where $m_{i j}=m_{i}+m_{j}$ and $i=1,2,3$. Each region, in turn, contains eight subregions according to the parities of $m_{i}-n_{i}$. For all subregions, we must check whether the corresponding three-point function is analytic, check the fusion rules, calculate the second-order counterterms, and finally compare with the results of the continuous approach.

Even this partial analysis reveals the following problems. As we saw, it turns out that only a special part of the selection rules can be satisfied using the resonance transformations. Hence, the selection rules of minimal models become modified after coupling to Liouville gravity. The nature of this phenomenon is not yet clear. It is natural to assume that a possible modification of the method is to require that satisfying this special part is a necessary condition. Indeed, the selection rules uniquely fix the form of the counterterms 
arising on a given level in the nonphysical region. On higher levels, these counterterms already enter the expressions for the correlators in the physical region, i.e., in the region where the fusion rules are not violated, and must therefore coincide with the results in the continuous approach. We plan to check this conjecture in the near future.

Another interesting question is to explain the nature of the resonance transformations from the standpoint of the Frobenius manifold structure. We believe that answering this question may help in finding a possible modification of the $A_{q}$ Frobenius manifold such that this modified version would be connected to MLG without using the resonance transformations.

\section{Acknowledgments}

I am grateful to A. Belavin, B. Dubrovin, and Yu. Rud for the useful discussions. I thank Professor K. Narain for the hospitality during my visit to ICTP in 2014 and the organizers of the 4th Workshop on Geometric Correspondences of Gauge Theories at SISSA. The study of the aspects of MLG theory connected with Frobenius manifolds was supported in part by the Russian Foundation for Basic Research (Grant No. 13-01-90614). The study of the form of the resonance transformations and the computation of the correlation functions was performed with the support of Russian Science Foundation (Grant No. 14-12-01383).

\section{A Details of the computations of the structure constants}

We start with a few comments on the multiplication law on the cotangent bundle in the initial coordinates $u^{i}$,

$$
d u^{i} \cdot d u^{j}=\widetilde{C}_{k}^{i j}(u) d u^{k}
$$

On the Frobenius manifolds $A_{q}$, we can construct so-called canonical ${ }^{10}$ coordinates $w^{i}$ such that the metric is diagonal (but not constant) in $w^{i}$. Multiplication of the tangent vectors in the canonical coordinates has the simple form

$$
\frac{\partial}{\partial w^{i}} \cdot \frac{\partial}{\partial w^{j}}=\delta_{i j} \frac{\partial}{\partial w^{i}}
$$

On the cotangent space, if we define

$$
d Q(z)=d u_{1} z^{q-2}+d u_{2} z^{q-3}+\cdots+d u_{q-1}
$$

using canonical coordinates, then we can easily verify the useful multiplication law property

$$
d Q(y) \cdot d Q(z)=\frac{Q^{\prime}(y) d Q(z)-Q^{\prime}(z) d Q(y)}{y-z} .
$$

In the left-hand side of (A.4), we have

$$
\sum_{m, n=0}^{q} d u_{m-1} \cdot d u_{n-1} y^{q-m} z^{q-n},
$$

\footnotetext{
${ }^{10}$ We note that there are three natural choices on the Frobenius manifold, initial, flat, and canonical coordinates, and each has its own advantages.
} 
and in the right-hand side, we have

$$
\frac{1}{y-z} \sum_{m, n=0}^{q}(q-m) u_{m-1} d u_{n-1}\left[y^{q-m-1} z^{q-n}-z^{q-m-1} y^{q-n}\right] .
$$

The expression in brackets can now be written as

$$
[\ldots]= \begin{cases}(y-z) y^{q-m-2} z^{q-n} \sum_{k=0}^{n-m-2}\left(\frac{z}{y}\right)^{k}, & n-m \geq 2 \\ 0, & n-m=1 \\ (y-z) y^{q-n-1} z^{q-m-1} \sum_{k=0}^{m-n}\left(\frac{z}{y}\right)^{k}, & n-m \leq 0 .\end{cases}
$$

Hence, the right-hand side in (A.4) becomes

$$
\begin{gathered}
\sum_{m, n=0}^{q}(q-m) u_{m-1} d u_{n-1}\left[\theta(n-m-2) \sum_{k=0}^{n-m-2} y^{q-m-k-2} z^{q-n+k}-\right. \\
\left.\theta(m-n) \sum_{k=0}^{m-n} y^{q-n-k-1} z^{q-m+k-1}\right],
\end{gathered}
$$

where $\theta(x)=1$ if $x \geq 0$ and 0 otherwise. Collecting the terms $y^{i} z^{j}$ in (A.4), we obtain ${ }^{11}$ the answer [25]

$$
\widetilde{C}_{i}^{j k}=(q+i-j-k+1) u_{j+k-i-2} \Theta(i, j, k)
$$

where we introduce the function

$$
\Theta(i, j, k)=\left\{\begin{aligned}
1 & \text { if } j, k \leq i \text { and } j+k>i \\
-1 & \text { if } j, k>i \text { and } j+k \leq i+q \\
0 & \text { otherwise }
\end{aligned}\right.
$$

and $u_{-1}=1, u_{0}=0$. On the other hand, the metric in flat coordinates is simple, and lowering an index $\alpha$ is just replacing it with $q-\alpha$. Hence,

$$
C_{\alpha \beta \gamma}=\frac{\partial v^{q-\alpha}}{\partial u^{i}} \frac{\partial v^{q-\beta}}{\partial u^{j}} \frac{\partial u^{k}}{\partial v^{\gamma}} \widetilde{C}_{k}^{i j}
$$

We can write the expansion in the vicinity $\mathbf{v}_{*}\left(\delta \mathbf{v}=\mathbf{v}-\mathbf{v}_{*}\right)$ :

$$
\begin{aligned}
& \frac{\partial u^{k}}{\partial v^{\gamma}}(\mathbf{v})=U_{\gamma}^{k}+\delta v^{\rho} U_{\rho \gamma}^{\prime k}+\ldots, \\
& \frac{\partial v^{\gamma}}{\partial u^{k}}(\mathbf{v})=V_{k}^{\gamma}+\delta v^{\rho} V_{\rho k}^{\prime \gamma}+\ldots,
\end{aligned}
$$

\footnotetext{
${ }^{11}$ I am grateful to Boris Dubrovin for the explanation regarding this derivation.
} 
where the coefficients can be found in terms of binomial coefficients,

$$
\begin{aligned}
U_{\gamma}^{k} & =\left(\begin{array}{c}
\frac{\gamma-k+q-2}{2} \\
\frac{\gamma+k-q}{2}
\end{array}\right)\left(\frac{v_{1}}{2}\right)^{\frac{\gamma+k-q}{2}} & \text { if } & \frac{\gamma+k-q}{2} \in \mathbb{N}, \\
V_{j}^{\rho} & =\frac{2 \rho}{q+\rho-j}\left(\begin{array}{c}
q-j-1 \\
\frac{q-\rho-j}{2}
\end{array}\right)\left(-\frac{v_{1}}{2}\right)^{\frac{q-\rho-j}{2}} & \text { if } & \frac{q-\rho-j}{2} \in \mathbb{N}, \\
U_{\alpha \beta}^{\prime k} & =\frac{q-k}{q}\left(\begin{array}{c}
\frac{\alpha+\beta-k-1}{2} \\
q-k
\end{array}\right)\left(\frac{v_{1}}{2}\right)^{\frac{k+\alpha+\beta-2 q-1}{2}} & \text { if } & \frac{k+\alpha+\beta-2 q-1}{2} \in \mathbb{N}, \\
V_{\beta k}^{\prime \alpha} & =-\frac{\alpha}{q}\left(\begin{array}{c}
q-1-k \\
\frac{\beta-\alpha-k-1}{2}
\end{array}\right)\left(-\frac{v_{1}}{2}\right)^{\frac{\beta-\alpha-k-1}{2}} & \text { if } & \frac{\beta-\alpha-k-1}{2} \in \mathbb{N} .
\end{aligned}
$$

In (A.14)-(A.17), if the conditions are not satisfied, then the corresponding values are equal to zero.

In particular, from (A.14), we obtain $\mathbf{u}\left(\mathbf{v}_{*}\right)$,

$$
u^{k}=\frac{2 q}{k+1}\left(\begin{array}{c}
\frac{2 q-k-1}{2} \\
\frac{k-1}{2}
\end{array}\right)\left(\frac{v_{1}}{2}\right)^{\frac{k+1}{2}} \text { if } \frac{k+1}{2} \in \mathbb{N} .
$$

In this notation, we have

$$
\begin{aligned}
& C_{\alpha \beta \gamma}\left(\mathbf{v}_{*}\right)=V_{i}^{q-\alpha} V_{j}^{q-\beta} U_{\gamma}^{k} \widetilde{C}_{k}^{i j}, \\
& \partial_{\rho} C_{\alpha \beta \gamma}\left(\mathbf{v}_{*}\right)= \\
& V_{\rho i}^{\prime q-\alpha} V_{j}^{q-\beta} U_{\gamma}^{k} \widetilde{C}_{k}^{i j}+V_{i}^{q-\alpha} V_{\rho j}^{\prime q-\beta} U_{\gamma}^{k} \widetilde{C}_{k}^{i j}+V_{i}^{q-\alpha} V_{j}^{q-\beta} U_{\rho \gamma}^{\prime k} \widetilde{C}_{k}^{i j}+V_{i}^{q-\alpha} V_{j}^{q-\beta} U_{\gamma}^{k} \tilde{C}_{\rho k}^{\prime i j}
\end{aligned}
$$

Some manipulations with the binomial coefficients give (2.16) and (2.17).

\section{B Some properties of the Jacobi polynomials}

The polynomials $P_{n}^{(0, b)}(t)$ satisfy the orthogonality condition

$$
\int_{-1}^{1} d t(1+t)^{b} P_{n}^{(0, b)}(t) P_{m}^{(0, b)}(t)=\frac{2^{b+1}}{2 n+b+1} \delta_{m, n} .
$$

In the standard normalization, $P_{n}^{(0, b)}(1)=1$, and the highest coefficient is

$$
P_{n}^{(0, b)}(t)=\frac{(b+n+1)_{n}}{n !}\left(\frac{t}{2}\right)^{n}+\ldots
$$

The Jacobi orthogonal polynomials $P_{n}^{(0, b)}(t)$ are normalized such that $P_{n}^{(0, b)}(1)=1$.

Below, we list properties of the Jacobi polynomials that reveal a more transparent structure of the second-order counterterms:

$$
\begin{aligned}
\frac{d}{d t} P_{n}^{(0, b)}(t) & =\frac{1}{2} \sum_{k=0}^{n-1}(2 k+b+2) P_{k}^{(0, b+1)}(t), \\
\frac{d}{d t}\left[(1+t)^{b+1} P_{n}^{(0, b+1)}(t)\right] & =(1+t)^{b} \sum_{k=0}^{n}(2 k+b+1) P_{k}^{(0, b)}(t),
\end{aligned}
$$


where the second equation can be derived from the first using integration by parts. Another useful property of the Jacobi polynomials is

$$
\frac{d}{d t} P_{n}^{(0, b)}(t)=\frac{b+n+1}{2} P_{n-1}^{(1, b+1)}(t) .
$$

\section{Details of the calculation of the four-point correlator}

Third derivatives of the action. We first discuss the third derivative $S_{\rho \sigma \eta}^{(0)}$. Using the definition and taking our basic recursion into account, we obtain

$$
\frac{\partial^{3} S^{(0)}}{\partial v_{\rho} \partial v_{\alpha} \partial v_{\beta}}=-\frac{1}{c_{1,2}} \frac{\partial^{3} \theta_{1,2}}{\partial v_{\rho} \partial v_{\alpha} \partial v_{\beta}}=-\frac{1}{c_{1,2}}\left(v_{1} \frac{\partial}{\partial v_{\rho}} C_{q-1}^{\alpha \beta}+C_{q-\rho}^{\alpha \beta}\right) .
$$

The WDVV requirement for the structure constants of the Frobenius algebra (which can be easily verified in our case) gives

$$
\frac{\partial}{\partial v_{\alpha}} C_{q-1}^{\gamma \beta}=\frac{\partial}{\partial v_{1}} C_{q-\gamma}^{\alpha \beta} .
$$

With this result, it is easy to find

$$
\frac{\partial^{3} S^{(0)}}{\partial v_{\rho} \partial v_{\alpha} \partial v_{\beta}}=-\frac{1}{c_{1,2}} \frac{\partial}{\partial v_{1}}\left(v_{1} C_{q-\rho}^{\alpha \beta}\right) .
$$

Because we are left with only the derivative with respect to $v_{1}$, we can now set $v_{k>1}=0$ and use (2.16) for the structure constant. After lowering the indices, we obtain expression (7.8).

Second derivatives of the first-order counterterms. We now discuss the second derivative $S_{\alpha \beta}^{(i)}$. We can split the calculation schematically into two parts. First, we find

$$
\frac{\partial^{2} S^{(i)}}{\partial v^{\alpha} \partial v^{\beta}}=\sum_{\gamma=1}^{q-1} C_{\alpha \beta \gamma}\left(-\frac{v_{1}}{q}\right)^{1-\gamma} \frac{\partial^{2} S^{(i)}}{\partial v_{1} \partial v^{\gamma}} .
$$

We then use the same trick as for $S_{\beta \rho \eta}^{(0)}$ : we set $v_{k>1}=0$ and use the explicit form of $S_{\gamma}^{(i)}$ in terms of Jacobi polynomials (5.3). Taking (B.4) into account, we obtain

$$
\frac{d}{d t} P_{k}^{(0, b)}(1)=\frac{(b+k+1) k}{2} .
$$

We now prove (C.4). Using the explicit form of the first-order counterterms for even $m-n$, we write the expansion in terms of Jacobi polynomials,

$$
\frac{\partial S^{(m n)}}{\partial v_{\alpha}}=\delta_{m, \alpha} \widetilde{N}_{m n} \sum_{k=0}^{\frac{m-n}{2}} b_{\frac{m-n}{2}-k}\left(-\frac{v_{1}}{q}\right)^{\left(\frac{m-n}{2}-k\right) q},
$$

where $b_{k}$ are expressed in terms of the coefficients of the Jacobi polynomials. Here, we prefer to absorb irrelevant factors in (5.3) in the normalization $\widetilde{N}_{m n}$. With the results in Lemma 3.2 taken into account, it then follows that

$$
S^{(m n)}=\sum_{k=0}^{\frac{m-n}{2}} \widetilde{A}_{m, m-n-2 k} \theta_{m, m-n-2 k},
$$


where

$$
\widetilde{A}_{m, m-n-2 k} x_{m, m-n-2 k}=\widetilde{N}_{m n} b_{\frac{m-n}{2}-k} .
$$

Differentiating (C.7) twice, we obtain

$$
\begin{aligned}
\frac{\partial^{2} S^{(m n)}}{\partial v_{\alpha} \partial v_{\beta}} & =C_{q-m}^{\alpha \beta} \sum_{k=0}^{\frac{m-n}{2}} \widetilde{A}_{m, m-n-2 k} \frac{\partial \theta_{m, m-n-2 k-1}}{\partial v_{q-m}} \\
& =C_{q-m}^{\alpha \beta}\left(-\frac{v_{1}}{q}\right)^{m} \widetilde{N}_{m n} \sum_{k=0}^{\frac{m-n}{2}} b_{\frac{m-n}{2}-k} \frac{y_{m, m-n-2 k-1}}{x_{m, m-n-2 k}}\left(-\frac{v_{1}}{q}\right)^{\frac{m-n-2 k-2}{2} q} .
\end{aligned}
$$

From (4.2), we derive

$$
\frac{y_{m, m-n-2 k-1}}{x_{m, m-n-2 k}}=\frac{m-n-2 k}{2} .
$$

Hence, on the line $v_{k>1}=0$, we have

$$
\frac{\partial^{2} S^{(m n)}}{\partial v_{\alpha} \partial v_{\beta}}=C_{q-m}^{\alpha \beta}\left(-\frac{v_{1}}{q}\right)^{m-q} \widetilde{N}_{m n} \sum_{k=0}^{\frac{m-n}{2}} b_{\frac{m-n}{2}-k}\left(\frac{m-n}{2}-k\right)\left(-\frac{v_{1}}{q}\right)^{\left(\frac{m-n}{2}-k\right) q}
$$

Comparing this expression with (C.6), we find

$$
\frac{\partial^{2} S^{(m n)}}{\partial v_{\alpha} \partial v_{\beta}}=C_{q-m}^{\alpha \beta}\left(-\frac{v_{1}}{q}\right)^{1-(q-m)} \frac{\partial^{2} S^{(m n)}}{\partial v_{1} \partial v_{m}} .
$$

A similar consideration can be performed for odd $m-n$ with the difference that instead of (C.10), we use

$$
\frac{x_{m, m-n-2 k-1}}{y_{m, m-n-2 k}}=\frac{m}{q}+\frac{m-n-2 k-1}{2} .
$$

The results of both calculations, for both even and odd $m-n$, gives (C.4).

Products of two structure constants. We can rewrite (7.11), (7.12), and (7.13) in the forms

$$
\begin{array}{r}
F_{1}(\chi, \xi, \mu, \nu)=\sum_{\gamma \in R(\chi, \xi) \cap R(\mu, \nu)} 1, \\
F_{2}(\chi, \xi, \mu, \nu)=\sum_{\gamma \in R(\chi, \xi) \cap \widetilde{R}(\mu, \nu)} 1, \\
F_{3}(\chi, \xi, \mu, \nu)=\sum_{\gamma \in R(\chi, \xi) \cap R(\mu, \nu)} \gamma,
\end{array}
$$

where

$$
\begin{aligned}
& R(\chi, \xi)=[|\chi+\xi-q|+1: 2: q-1-|\chi-\xi|], \\
& \widetilde{R}(\mu, \nu)=[|\mu-\nu|+1: 2: q-|\mu+\nu-q|-1] .
\end{aligned}
$$


Hence,

$$
\begin{aligned}
& R(\chi, \xi) \cap R(\mu, \nu)=\left[A_{1}, B_{1}\right], \\
& R(\chi, \xi) \cap \widetilde{R}(\mu, \nu)=\left[A_{2}, B_{2}\right],
\end{aligned}
$$

where

$$
\begin{aligned}
& A_{1}=\max (|\chi+\xi-q|+1,|\mu+\nu-q|+1), \\
& B_{1}=\min (q-|\chi-\xi|-1, q-|\mu-\nu|-1), \\
& A_{2}=\max (|\chi+\xi-q|+1,|\mu-\nu|+1), \\
& B_{2}=\min (q-1-|\chi-\xi|, q-1-|\mu+\nu-q|),
\end{aligned}
$$

or

$$
\begin{aligned}
& A_{1}=\frac{1}{2}(|\chi+\xi-q|+|\mu+\nu-q|+2+|| \chi+\xi-q|-| \mu+\nu-q||), \\
& B_{1}=\frac{2 q-2-|\chi-\xi|-|\mu-\nu|}{2}-\frac{|| \chi-\xi|-| \mu-\nu||}{2}, \\
& A_{2}=\frac{1}{2}(|\chi+\xi-q|+|\mu-\nu|+2+|| \chi+\xi-q|-| \mu-\nu||), \\
& B_{2}=\frac{1}{2}(2 q-2-|\chi-\xi|-|\mu+\nu-q|-|| \chi-\xi|-| \mu+\nu-q||) .
\end{aligned}
$$

After replacing $\gamma=A_{1,2}+2 s$, we have

$$
\begin{aligned}
& F_{1}(\chi, \xi, \mu, \nu)=\sum_{\substack{s=0 \\
B_{2}-A_{2}}}^{\frac{B_{1}-A_{1}}{2}} 1=\frac{B_{1}-A_{1}}{2}+1, \\
& F_{2}(\chi, \xi, \mu, \nu)=\sum_{s=0}^{\substack{B_{1}-A_{1} \\
2}} 1=\frac{B_{2}-A_{2}}{2}+1, \\
& F_{3}(\chi, \xi, \mu, \nu)=\sum_{s=0}\left(A_{1}+2 s\right)=\frac{B_{1}+A_{1}}{2}\left(\frac{B_{1}-A_{1}}{2}+1\right) .
\end{aligned}
$$

These results lead to (7.14), (7.15), and (7.16).

Open Access. This article is distributed under the terms of the Creative Commons Attribution License (CC-BY 4.0), which permits any use, distribution and reproduction in any medium, provided the original author(s) and source are credited.

\section{References}

[1] A.M. Polyakov, Quantum Geometry of Bosonic Strings, Phys. Lett. B 103 (1981) 207 [INSPIRE].

[2] A.A. Belavin, A.M. Polyakov and A.B. Zamolodchikov, Infinite Conformal Symmetry in Two-Dimensional Quantum Field Theory, Nucl. Phys. B 241 (1984) 333 [INSPIRE]. 
[3] A.B. Zamolodchikov, Three-point function in minimal Liouville gravity, Theor. Math. Phys. 142 (2005) 183 [hep-th/0505063] [INSPIRE].

[4] A. Belavin and A. Zamolodchikov, Integrals over moduli spaces, ground ring, and four-point function in minimal Liouville gravity, Theor. Math. Phys 147 (2006) 729 [hep-th/0510214] [INSPIRE].

[5] A. Zamolodchikov, Gravitational Yang-Lee Model: Four Point Function, Theor. Math. Phys. 151 (2007) 439 [hep-th/0604158] [INSPIRE].

[6] V. Belavin, Torus Amplitudes in Minimal Liouville Gravity and Matrix Models, Phys. Lett. B 698 (2011) 86 [arXiv:1010.5508] [INSPIRE].

[7] V.A. Kazakov, A.A. Migdal and I.K. Kostov, Critical Properties of Randomly Triangulated Planar Random Surfaces, Phys. Lett. B 157 (1985) 295 [inSPIRE].

[8] V.A. Kazakov, Ising model on a dynamical planar random lattice: Exact solution, Phys. Lett. A 119 (1986) 140 [InSPIRE].

[9] V.A. Kazakov, The Appearance of Matter Fields from Quantum Fluctuations of 2D Gravity, Mod. Phys. Lett. A 4 (1989) 2125 [INSPIRE].

[10] M. Staudacher, The Yang-lee Edge Singularity on a Dynamical Planar Random Surface, Nucl. Phys. B 336 (1990) 349 [inSPIRE].

[11] E. Brézin and V.A. Kazakov, Exactly Solvable Field Theories of Closed Strings, Phys. Lett. B 236 (1990) 144 [InSPIRE].

[12] M.R. Douglas and S.H. Shenker, Strings in Less Than One-Dimension, Nucl. Phys. B 335 (1990) 635 [inSPIRE].

[13] D.J. Gross and A.A. Migdal, Nonperturbative Two-Dimensional Quantum Gravity, Phys. Rev. Lett. 64 (1990) 127 [INSPIRE].

[14] M.R. Douglas, Strings in Less Than One-dimension and the Generalized $K^{-} D^{-} V$ Hierarchies, Phys. Lett. B 238 (1990) 176 [InSPIRE].

[15] I.K. Kostov, Strings with discrete target space, Nucl. Phys. B 376 (1992) 539 [hep-th/9112059] [INSPIRE].

[16] I.K. Kostov, Gauge invariant matrix model for the A-D-E closed strings, Phys. Lett. B 297 (1992) 74 [hep-th/9208053] [INSPIRE].

[17] V.G. Knizhnik, A.M. Polyakov and A.B. Zamolodchikov, Fractal Structure of $2 D$ Quantum Gravity, Mod. Phys. Lett. A 3 (1988) 819 [INSPIRE].

[18] G.W. Moore, N. Seiberg and M. Staudacher, From loops to states in $2-D$ quantum gravity, Nucl. Phys. B 362 (1991) 665 [inSPIRE].

[19] I.K. Kostov and V.B. Petkova, Non-rational $2-D$ quantum gravity. I. World sheet CFT, Nucl. Phys. B 770 (2007) 273 [hep-th/0512346] [INSPIRE].

[20] I.K. Kostov and V.B. Petkova, Non-Rational 2D Quantum Gravity II. Target Space CFT, Nucl. Phys. B 769 (2007) 175 [hep-th/0609020] [INSPIRE].

[21] I.K. Kostov and V.B. Petkova, Bulk correlation functions in $2-D$ quantum gravity, Theor. Math. Phys. 146 (2006) 108 [hep-th/0505078] [INSPIRE].

[22] A.A. Belavin and A.B. Zamolodchikov, On Correlation Numbers in 2D Minimal Gravity and Matrix Models, J. Phys. A 42 (2009) 304004 [arXiv:0811.0450] [InSPIRE]. 
[23] A. Belavin, B. Dubrovin and B. Mukhametzhanov, Minimal Liouville Gravity correlation numbers from Douglas string equation, JHEP 01 (2014) 156 [arXiv:1310.5659] [INSPIRE].

[24] A.A. Belavin and V.A. Belavin, Frobenius manifolds, Integrable Hierarchies and Minimal Liouville Gravity, JHEP 09 (2014) 151 [arXiv:1406.6661] [INSPIRE].

[25] V. Belavin, Unitary Minimal Liouville Gravity and Frobenius Manifolds, JHEP 07 (2014) 129 [arXiv: 1405.4468] [INSPIRE].

[26] L. Spodyneiko, Minimal Liouville Gravity on the Torus via Matrix Models, arXiv: 1407.3546 [INSPIRE].

[27] I. Krichever, The Dispersionless Lax equations and topological minimal models, Commun. Math. Phys. 143 (1992) 415.

[28] B. Dubrovin, Integrable systems in topological field theory, Nucl. Phys. B 379 (1992) 627 [INSPIRE].

[29] R. Dijkgraaf, H.L. Verlinde and E.P. Verlinde, Topological strings in $d<1$, Nucl. Phys. B 352 (1991) 59 [INSPIRE].

[30] P.H. Ginsparg, M. Goulian, M.R. Plesser and J. Zinn-Justin, $(p, q)$ string actions, Nucl. Phys. B 342 (1990) 539 [InSPIRE].

[31] P. Di Francesco and D. Kutasov, Unitary minimal models coupled to $2-D$ quantum gravity, Nucl. Phys. B 342 (1990) 589 [inSPIRE]. 